\title{
E-learning through telemedicine in neurosurgical teaching and patient care
}

\author{
Sushil K. Meher* \\ Department of Computer Facility, \\ All India Institute of Medical Sciences, \\ Ansari Nagar, 110029, New Delhi, India \\ Email: s_meher@hotmail.com \\ *Corresponding author
}

\section{Nilesh S. Kurwal and Ashish Suri}

Department of Neurosurgery,

All India Institute of Medical Sciences,

Ansari Nagar, 110029, New Delhi, India

Email: nileshkurwal@gmail.com

Email: surineuro@hotmail.com

\begin{abstract}
Neurosurgery is both an art and a science. The art of neurosurgery can be learnt by working with a Senior Neurosurgeon, over a period of time. But the science of neurosurgery has to be learnt from intense reading of textbooks, journals and elucidation of difficult topics by a masterly teacher. Many of the concepts in neurosurgery and applied basic sciences can be better learnt with the help of a good teacher of neurosurgery. Much of the clinical acumen is gained at the bedside teaching by a good teacher. But unfortunately, the species of dedicated teachers of neurosurgery seems to be fast dwindling. So the technology can provide the gap of these problems. We can use the telemedicine technology and make a schedule teaching program from a good neurosurgical specialist.
\end{abstract}

Keywords: telemedicine; integrated service digital network; ISDN; very small aperture terminal; VSAT; continuing medical education; CME.

Reference to this paper should be made as follows: Meher, S.K., Kurwal, N.S. and Suri, A. (2017) 'E-learning through telemedicine in neurosurgical teaching and patient care', Int. J. Telemedicine and Clinical Practices, Vol. 2, No. 1, pp.2-11.

Biographical notes: Sushil K. Meher holds an MCA (NIT, Rourkela, Orissa), MBA (Hospital Management), MPhil (CS), PhD (eHealth) and is a Sr. Medical Informatics Specialist at AIIMS (all India Institute of Medical Sciences), New Delhi, India for last 23 years. He is recognised as a Leader in Hospital Digitisation and Biomedical Informatics that provides scientific foundation for models of decision-making in health care and for medical education. His research interests include competent performance in the workplace, team decision-making, processing re-engineering, clinical research informatics, telehealth and mobile health, public health informatics, PACS, patient safety and medical errors, human-computer interaction in health-care domains. He also actively involved IT implementation in AIIMS. 
Nilesh S. Kurwal is Medical graduate from AFMC Pune, and trained in Medical Informatics from AIIMS New Delhi. He have hold various important posts in Government of India including Joint Director (Med Informatics) at Min of Defence Government of India. Presently, holding post of DA Director of Med Services at DANTAK in Bhutan. He have planned and implemented various important health IT and telemedicine projects for Government of India.

This paper is a revised and expanded version of a paper entitled 'E-learning through telemedicine in neurosurgical teaching and patient care' presented at International Conference on Intelligent Computing, Communication and Convergence (ICCC 2014), Bhubaneswar, Odisa, India, 27-28 December 2014.

\section{Introduction}

Telemedicine has the potential to revolutionise the delivery of modern and advanced health care to both rural and urban populations. In many parts of the world this information technology tool has proved of great utility in saving priceless human lives as well as delivering information to remote areas where tertiary care was not available (Baquet, 1997; LaMay, 1997; Ganapathy, 2002). Besides patient care, telemedicine also proved to be of great help in knowledge transfer and education for health providers in rural and remote areas where formal education means are proving of little help (Baquet, 1997; Ganapathy, 2002).

Role of telemedicine has already been evaluated and demonstrated in neurology clinics by Finley et al. (2001) and Misra et al. (2004) in Sanjay Gandhi Postgraduate Institute (SGPGI), Lucknow. In many parts of world, including India, telemedicine has been utilised in different specialties of medicine like cardiology, neurology, surgery, and dermatology, for teleconsultations on patient issues as well as education of residents as well as practitioners; however its role in neurosurgical educations has not been explored much. In India, Kapoor et al. (2005) have revolutionised head trauma management by setting a complete network of mobile telemedicine centres, as well as teleconsultations, but the great potential of telemedicine in the field of resident training and education of practicing neurosurgeons still remains largely unexplored. In this article, we review the role of telemedicine as an educational tool in neurosurgical practice, and explore the role of e-learning in neurosurgical teaching and training.

\section{Historical background}

Telemedicine is closely related to telecommunication. It has been defined as delivery of healthcare services to the underserved, employing telecommunication means like integrated service digital network (ISDN), very small aperture terminal (VSAT), Wi-Max, 3G, internet or through ordinary telephone lines (LaMay, 1997). Telemedicine can be of two types:

1 synchronous or real time

2 asynchronous or store-and-forward type. 
Clinical branches, like neurology, cardiology etc utilises synchronous type where the patient and physician sit in a same chamber with direct talk while disciplines like pathology and radiology can utilise asynchronous methods with more utility, where photographs/films are first stored and sent for opinion in office time (http://en.wikipedia.org/wiki/Telemedicine).

Telemedicine as a tool of medical education was first utilised in delivering the continuing medical education (CME) program for physicians in rural areas by Ricci et al. (2005). They found that though 'in-person CME' was rated better than 'telemedicine CME', it can be a good delivery tool with only $18 \%$ of participants stating that they would have preferred to attend the same CME in person. $60 \%$ of participants stated that it was as effective as 'in-person CME'.

Telemedicine was utilised in training of two surgeons for endocrine surgeries from a tertiary care centres and found that at the end of training, the mortality and morbidity of the procedures carried out by the trainees were same as that of the parent training institute, thus ushering it as a potential means of direct surgical education in surgical branches (Pradeep et al., 2007). Surgical images can be transferred and advice can be obtained from a distance, as shown by J.D. Westwood (Gandsas et al., 2004), who demonstrated the utility of telemedicine in successfully transmitting the endoscopic surgeries between Hanyang University Hospital in Seoul and Kyushu University Hospital in Japan. Other models for surgical training have also been tried, like a laparoscopic splenectomy transmitted live to eight handheld computers simultaneously through wireless network (Naidu, 2003). All eight viewers were able to view the procedure and to hear the surgeon's comments throughout the entire duration of the operation, which is a useful opportunity for residents and trainees to learn.

\section{What is e-learning?}

E-learning commonly refers to the intentional use of networked information and communications technology in teaching and learning (BabyManoj, 2005). E-learning is four types which are self explanatory.

1 individualised, self paced online e-learning

2 individualise, self paced offline e-learning

3 group-based e-learning synchronously

4 group-based e-learning asynchronously.

The growing interest in e-learning seems to be coming from the various organisations which are offering the distance education programs. The key attributes to the success and growth of e-learning remains its ability to enable flexible access to information and resources at a time, place, and pace that is suitable for individual learners rather than teacher or organisations. However, the main obstacle remains the lack of access to the necessary technology and infrastructure. The goal of e-learning for distance education is mainly is to provide access to the subject matter content and learning resources via networked information technologies across the range of settings such as conventional classrooms, workplaces, and homes at a time, place and pace convenient to students (Sood and Bhatia, 2005). E-learning can be practised through many modalities like 
e-mails, e-mail lists, inter-relay chats, electronic bulletin boards, and computer conferencing depending on whether it is one to one or one to many communication systems, many to many. Computer conferencing offers a good two way communication and is being utilised in medical fields.

A good conferencing system should have separate conferences for broad topics, threaded discussions within the conference, informative topic lists giving concerned details about topics of discussion with proper and defined access to the concerned participants. At present e-learning is delivered through online learning management systems (LMS) which are software applications that comprise a suite of tools for learning and teaching online. Some, such examples are WebCT, Blackboard, Moodle etc. These LMS should be easier to use, more robust, scalable and more easily customisable. These LMS works with digital learning objects (DLO) which are the specially designed learning electronic objects which are discrete in nature and can be categorised, and stored independently and reused in a range of educational settings. From the above discussion, it is clear that online learning environments are the result of a team effort which brings together a wide range of expertise including a subject matter expert, LMS, web developers, graphic artists, and system engineers. To conclude, e-learning is a complex undertaking preceded by careful planning requiring expertise in many disciplines. It is neither a cheap and easy educational option nor does it offer a quick fix for the problems.

\section{E-learning for neurosurgical training}

Neurosurgery is a vast discipline consisting of many subspecialties including vascular neurosurgery, trauma, epilepsy surgeries, and spinal surgeries. Complex surgeries in each of these specialties are generally performed at higher centres. E-learning can be a good medium for technical training and education of neurosurgeons across the country in these subspecialties. Synchronous and asynchronous, both types of e-learning can be used in surgical training. For example a module containing aneurysm surgery can be made accessible through the internet, asynchronously along with the demonstrations and difficulties can be discussed through e-mails. Similarly an operation can be directly transmitted and participant can be invited through teleconferencing offering a discussion about the technical details of the operations. Similarly, distance learning programs can also be run for neurosurgeons across the country or in fact world for technical assistance in surgeries and recent advances in the discipline. But as stated earlier, it is teamwork and need to plan and implemented carefully for optimal benefit to the residents as well as practitioners of neurosurgery.

\section{Present status of telemedicine in India}

The efficacy of telemedicine has already been shown through the network established by the Indian Space Research Organization (ISRO), which has connected 65 super-specialty hospitals with 200 rural and remote hospitals across the country through its geo-stationary satellites. This network has enabled thousands of patients in remote places such as Jammu and Kashmir, Andaman and Nicobar Islands, Lakshadweep Islands, and tribal areas of the central and northern eastern regions of India to gain access to consultations with experts in super-specialty medical institutions (Bagchi, 2006). 
There are several pilot projects which ISRO has undertaken to provide education in many fields other than health sector in different states of India. In Karnataka, the Visveswaraya Technological University has begun regular classes providing lessons for 100 engineering colleges from 2004. In Maharashtra, Y.B Chavan Open University is conducting regular contact classes in 75 locations spread over the State from October 2004. Similarly, in Madhya Pradesh, Rajiv Gandhi Technical University is conducting trial transmission of classes with 25 nodes. Use of telemedicine for the benefit of primary education is currently under implementation under the Primary Education Pilot Project (Karnataka) of 'Sarva Shiksha Abhiyan' covering about 885 primary and secondary schools (Indian Space Research Organization, 2005).

Educational teleconferencing between SGPGI, Lucknow and SCB Medical College, Cuttack, which are 1,500 km apart, was conducted to around 30 educational sessions, discussing interesting cases in neurology and concerned radiology. It was participated by faculty members and residents, and they found it a very useful learning and teaching experience (Misra et al., 2005, 2004). There are also reports from our country on the use of telemedicine in various other disciplines like pathology, dermatology, radiology, emergency medicine and disaster management in recent years. In their experience, Bhatia and Sharma (2006) found that good patient satisfaction can be achieved in ophthalmology practice thorough telemedicine in rural India. A cross-sectional survey among patients who attended a tele-ophthalmology screening conducted across eight villages in rural Tamilnadu found that patients were satisfied with the teleconsultations (Bhatia and Sharma, 2006). Baruah (2005) has practiced a telepathology and found good results with glass slide transfer and digital images of pathology specimens. He believes that acceptance of telepathology by practitioners is the main shortcoming Similarly, Desai et al. (2004) have practised telemedicine service between a tertiary cancer referral centre, Tata Memorial Centre and a sister concern in rural area viz. Nargis Dutt Memorial Cancer Hospital, Barshi, Solapur. They experienced a good efficacy in quality and time in reporting pathological specimens in which $99 \%$ diagnosis was given and major discrepancies occurred in only $6 \%$ of cases with one third of the reporting was done on the same day of receiving the specimens. Similar is the experience with tele-radiology, dermatology, and other specialties which are tried in India (Jankharia, 2001; Kanthraj and Srinivas, 2007). Telemedicine was used as effective mean of health care delivery and patient care in disaster management caused by cholera outbreak during the holy pilgrimage of Hindus in Maha Kumbh Mela in Prayag, Uttar Pradesh (Pradeep et al., 2006).

Ganapathy (2004) and Bai et al. (2007) have started teleconsultations and CME in various specialties in Apollo Hospital Chennai, connecting various other tertiary care centres and rural, suburban population. Apollo Hospital Chennai has started mobile telemedicine vans, which are equipped with all first aids and telemedicine facility. Patient is assessed on the spot and consultation is sought urgently through telemedicine with a consultant sitting in tertiary care centre and the patient is managed accordingly. But unlike its use in patient management, there are very few reports of utilisation of this facility for education and knowledge transfer between practitioners and higher tertiary care centres (Misra et al., 2004). 


\section{Telemedicine: potentially effective educational tool in neurosurgery}

In surgical disciplines, operation theatre training forms an important part of resident training program. Telemedicine can play an important role in this regard. Live surgeries can be transmitted using ISDN lines or through VSAT, and a good two-way communication can be established between the operating surgeon and students outside the operation theatre and even outside the city. Developments in telecommunication technologies have now made it possible to transfer good quality images through these means. With this, many students can discuss the technical details of the operation with the operating surgeon directly while the operation is in progress. This learning experience can be equally beneficial for practicing neurosurgeons that are not habitual with the complex surgeries generally performed at higher centres. The other way round is also possible. Advice or expert opinion can be taken from the senior surgeons based in higher centres, in case of difficulty encountered during a surgery at a remote place, especially if the issue is of decision making rather than technical difficulty during the surgery. However, some legal issues need to be sorted out before this system can be fully implemented as these are discussed in some detail below.

Similarly, seminars on different topics, which are a routine in the academic roster of tertiary care medical institutes like AIIMS, can be transmitted (live or recorded) to other centres for their benefit. Panel discussions, where the panel may consist of experts from various parts of India or in fact world, can be held on current topics and recent developments and discussion can be held between various centres through teleconferencing. These seminars and lectures by experts can also be helpful for medical undergraduates and post graduates aspiring to take up neurosurgery as a career. Telemedicine facility can be extended to interesting clinical case discussions which are often held at higher medical centres for resident training.

\section{How telemedicine is superior to traditional measures delivery of information}

Traditionally, conferences and workshops are organised yearly for updating practicing neurosurgeons and residents with newer advances in the field. However, they have time and place constraints and can be attended by a limited number of doctors at a time. Telemedicine can be a useful addition to these conferences as they are very effective as a mean of education.

Presently, internet is a good medium for knowledge transfer and updating with recent developments, but only one way communication is possible with internet and printed media education. Virtual surgical workshops and panel discussions can be held through the telemedicine facility which is proving more efficient, cost effective and convenient to medical practitioners across the country than traditional means. There are some reports from other countries that these conferences are being telecasted live and video conferencing is available during the sessions for discussion. Direct transmission from the operation theatre to the other linked centres made the actual discussion between operating surgeon and the observer possible during the operation. 


\section{AIIMS experience}

Telemedicine facility in AIIMS was inaugurated by the honourable health minister in January 2006. It was opened with the aim of providing specialised health care to the rural population in the Faridabad district of Haryana and to assist the medical practitioners at Ballabgarh CHRS. It has served the purpose over the last two years providing more than 500 teleconsultations in various specialties to rural populations. At present we are providing teleconsultations to Nagaland, and North East states of India regularly for Neurosurgery and other disciplines.

Telemedicine at AIIMS is also engaged in educational activities like CMEs, seminars, and Lectures through connections to various colleges across India. We also telecasted some conferences which were held at AIIMS in various disciplines across the India benefiting the medical practitioners all over the country. Effective use of telemedicine facility was done in 9th Annual Conference of the Skull Base Society of India and WFNS microsurgery and endoscopy skull base cadaveric workshop. Various informative sessions held during the conference were broadcasted across India.

\subsection{Adaveric dissection}

Cadaveric dissection of endoscopic transsphenoidal dissections followed by anterior and middle cranial fosse anatomy and various surgical approaches was directly done by 20 tutors and 80 hands - on participants in the dissection hall. This dissection was captured by advanced cameras and was transmitted to the auditorium at AIIMS along with Mumbai and Chennai. Discussions were held between the tutors in the dissection hall and spectators in the auditorium virtually giving one to one teaching experience to all the spectators.

Figure 1 Transmission process of Cadaveric skull base dissection hands on workshop (see online version for colours)

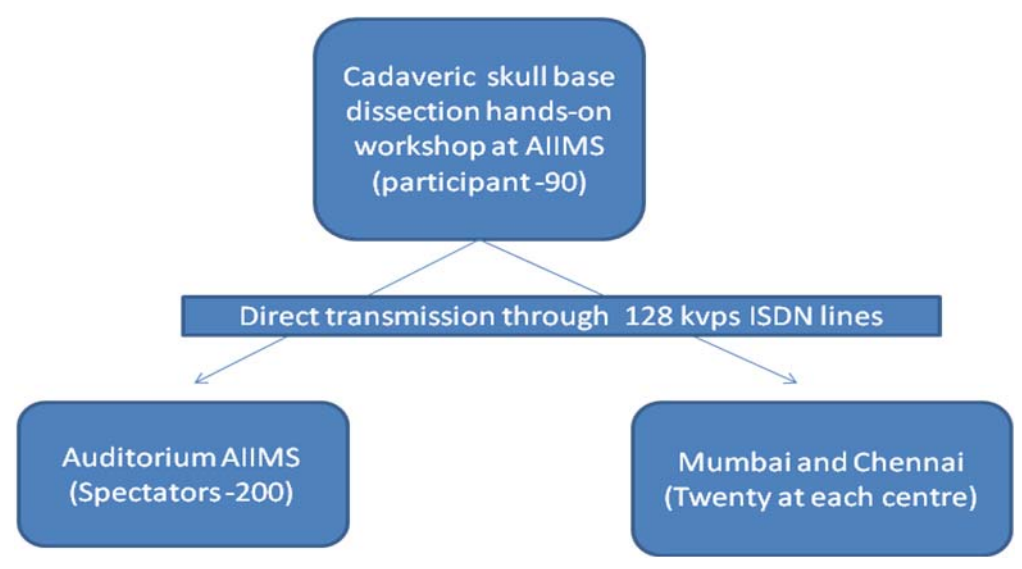

\subsection{Lecture and presentations}

Lecture and presentations were also transmitted through ISDN lines to these cities across India. The lecture on transpetrous approach to skull base lesions by Prof. T. Kawase from 
Tokyo, Japan was delivered in the auditorium, along with clarifications of various queries of spectators. This achieved a class teaching pattern through the telemedicine facility. It has explained more in Figure 2.

Figure 2 Standard procedure for transmission of E-learning through telemedicine in neurosurgical

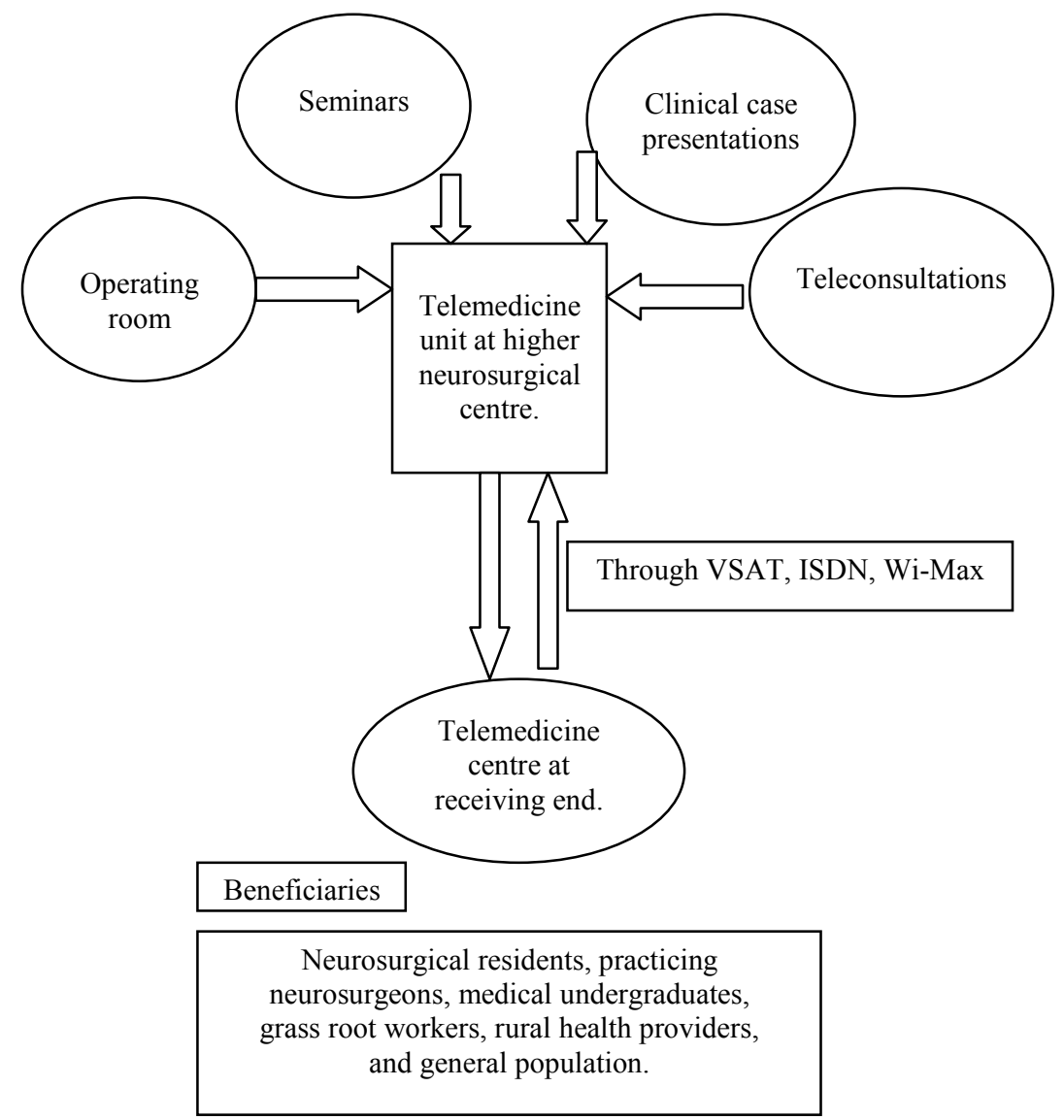

\subsection{Surgery transmission}

Live transmission of intracranial surgery from Pittsburg (Dr. Amin Kassam) was done along with direct discussion of important surgical steps and minor clarifications which was achieved thorough a dialogue between operating surgeon and spectators. This simulated the conventional one to one operation theatre teaching of surgical residents by faculties.

Telemedicine facility has helped us to deliver the core neurosurgical teaching to almost more than 500 neurosurgeons across the India, greatly increasing the efficacy of knowledge transfer. 


\section{Legal issues}

There appear to be four main areas of concern with the implementation of telemedicine and tele-consultations in clinical practice (Sood and Bhatia, 2005):

1 doctor-patient relationship

2 malpractice and cross-border licensure

3 standards of care different states

4 patient confidentiality

5 reimbursement issues.

Though a central medical licensing body exists in India, there are many medical degrees given by respective states and which are not considered valid in other states, so this cross border telemedicine can become a complex issue to handle. If a patient seeks medical advice from a doctor $\mathrm{X}$ in a neighbouring state, though his family physician $\mathrm{Y}$, who is not licensed in the state of the patient, who should be held responsible for the adverse outcome consequences to wrong decisions of the doctor $\mathrm{X}$ ?

Distance patient care has always been debated for responsibility of complications of treating physicians and surgeons. Questions have been raised in the past about the medico legal aspects of expert opinions and diagnosis offered by teleconsultations and compensation issues in case of default/neglect of duty by treating physician or surgeon (Sood and Bhatia, 2005). The issue of security of confidential patient information which is telecasted is also important, which if not maintained can be punishable under the court of law. Presently information technology scientists have started providing solutions to these security problems, but still it is not complete and not guarded by any legal system.

At present, there are no definite guidelines on the role of telemedicine in patient care or education in our country. A national task force for defining the role telemedicine was formed under the ministry of health and family welfare in March 2006, and it has formulated some guidelines for this purpose. However, presently there are no established standards in our country. Medico legal issues in teleconsultations and insurance reimbursements need to be looked into for effective implementation of this facility across the country. As far as education is concerned, legal issues have a very limited role, however the disclosure of confidential patient information to persons not concerned with treatment of patients can be of legal dispute, and hence needs to be standardised.

\section{References}

BabyManoj, R. (2005) ISRO to Set Up 100 More Telemedicine Centers Across the Country, Issue dated 16th to 31st March 2005 [online] http://archivepharma.financialexpress.com/20050317/ healthnews02.shtml.

Bagchi, S. (2006) 'Telemedicine in rural India', PLoS Med, 7 March, Vol. 3, No. 3, p.e82, Epub.

Bai, V.T., Murali, V., Kim, R. and Srivatsa, S.K. (2007) 'Teleophthalmology-based rural eye care in India', Telemed J. E Health, June, Vol. 13, No. 3, pp.313-321.

Baquet, C.R. (1997) 'An overview of telemedicine', J. Assoc. Acad. Minor Physicians, Vol. 8, No. $1, \mathrm{pp} .2-10$.

Baruah, M.K. (2005) 'The practice of telepathology in India', J Postgrad Med, October-December, Vol. 51, No. 4, pp.316-318. 
Bhatia, J.S. and Sharma, S. (2006) 'Telemedicine odyssey customised telemedicine solution for rural and remote areas in India', Stud Health Technol. Inform., Vol. 121, pp.22-35, PMID: 17095800 [PubMed - indexed for MEDLINE].

Desai, S., Patil, R., Kothari, A., Shet, T., Kane, S., Borges, A. and Chinoy, R. (2004) 'Static telepathology consultation service between Tata Memorial Centre, Mumbai and Nargis Dutt Memorial Charitable Hospital, Barshi, Solapur, Maharashtra: an analysis of the first 100 cases', Indian J. Pathol. Microbiol., October, Vol. 47, No. 4, pp.480-485.

Finley, J.P., Beland, M.J., Boutin, C., Duncan, W.J., Dyck, J.D., Hosking, M.C. and Nykanen, D. (2001) 'A national network for the tele-education of Canadian residents in pediatric cardiology', Cardiol. Young, September, Vol. 11, No. 5, pp.526-531.

Ganapathy, K. (2002) 'Telemedicine and neurosciences in developing countries', Surg. Neurol., December, Vol. 58, No. 6, pp.388-94.

Ganapathy, K. (2004) 'Role of telemedicine in neurosciences', Stud. Health Technol. Inform., Vol. 104, pp.116-124, PMID: 15747970 [PubMed - indexed for MEDLINE] [online] http://www.ncbi.nlm.nih.gov/entrez/eutils/elink.fcgi?dbfrom=pubmed\&retmode=ref\&cmd=prl inks\&id=22080284.

Gandsas, A., McIntire, K. and Park, A. (2004) 'Live broadcast of laparoscopic surgery to handheld computers', Surg Endosc, June, Vol. 18, No. 6, pp.997-1000.

http://en.wikipedia.org/wiki/Telemedicine (accessed 14 March 2013).

Indian Space Research Organization (2005) ISRO Annual Report 2004-2005: Space Applications [online] http://www.isro.org/rep2005/SpaceApplications.htm (accessed 14 March 2013)..

Jankharia, B. (2001) 'Current status and history of teleradiology in India', Int. J. Med. Inform., May, Vol. 61, Nos. 2-3, pp.163-166.

Kanthraj, G.R. and Srinivas, C.R. (2007) 'Store and forward teledermatology', Indian J. Dermatol. Venereol. Leprol., January-February, Vol. 73, No. 1, pp.5-12.

Kapoor, L., Mishra, S.K. and Singh, K. (2005) 'Telemedicine: experience at SGPGIMS, Lucknow', J. Postgrad. Med., October-December, Vol. 51, No. 4, pp.312-315.

LaMay, C.L. (1997) 'Telemedicine and competitive change in health care', Spine (Phila Pa), 1 January, Vol. 22, No. 1, pp.88-97.

Misra, U.K., Kalita, J., Mishra, S.K. and Yadav, R.K. (2004) 'Telemedicine for distance education in neurology: preliminary experience in India', J. Telemed. Telecare, Vol. 10, No. 6, pp.363-365.

Misra, U.K., Kalita, J., Mishra, S.K. and Yadav, R.K. (2005) 'Telemedicine in neurology: underutilized potential', Neurol. India, March, Vol. 53, No. 1, pp.27-31.

Naidu, S. (2003) E-learning: A Guidebook of Principles, Procedures and Practices, 2nd Revised ed., Pub. Commonwealth Educational Media Centre for Asia (CEMCA, 2006), First published in 2003, New Delhi, India.

Pradeep, P.V., Mishra, A., Mohanty, B.N., Mohapatra, K.C., Agarwal, G. and Mishra, S.K. (2007) 'Reinforcement of endocrine surgery training: impact of telemedicine technology in a developing country context', World J. Surg., August, Vol. 31, No. 8, pp.1665-1671.

Pradeep, P.V., Mishra, S.K., Vaidyanathan, S., Nair, C.G., Ramalingam, K. and Basnet, R. (2006) 'Telementoring in endocrine surgery: preliminary Indian experience', Telemed J. E Health, February, Vol. 12, No. 1, pp.73-77.

Ricci, M.A., Caputo, M.P., Callas, P.W. and Gagne, M. (2005) 'The use of telemedicine for delivering continuing medical education in rural communities', Telemed J. E Health, April, Vol. 11, No. 2, pp.124-129.

Sood, S.P. and Bhatia, J.S. (2005) 'Development of telemedicine technology in India: 'Sanjeevani' - an integrated telemedicine application', J. Postgrad. Med., October-December, Vol. 51, No. 4, pp.308-311. 